- Report on Experiments and Clinical Cases-

\title{
Analysis of Antimicrobial Resistance for Staphylococcus aureus Strains by WHONET 5 : Microbiology Laboratory Database Software
}

\author{
Toru Mochizuki ${ }^{1}$ Naoto Okamoto ${ }^{2}$, Teruko Yagishitaㄹ, Kitoji Takuhiro', Kunihiro Mashiko', \\ Futoshi Ogawa ${ }^{1}$, Naoki Tosaka ${ }^{1}$, Akira Kurokawa ${ }^{1}$ and Yasuhiro Yamamoto ${ }^{1}$ \\ ${ }^{1}$ Department of Emergency and Critical Care Medicine, Nippon Medical School \\ ${ }^{2}$ Department of Clinical Laboratory, Nippon Medical School
}

\begin{abstract}
Objectives: To analyze our hospital laboratory microbiological data by using WHONET 5-Microbiology laboratory database software-, and to acquire information about antimicrobial resistance of Staphylococcus aureus strains among every ward.

Materials and methods: The database of Staphylococcus aureus strains had been brought to our hospital microbiology laboratory from every ward in our hospital from September 2001 till December 2002. Analysis was performed under the condition as one isolate per one patient. Starting of 'resistance profile' analysis in WHONET 5 and analyzing the microbiological laboratory testing reports for every ward. We chose Oxacillin, Levofloxacin, Erythromycin and Gentamicin as the antimicrobials that need to be investigated for resistance. We evaluated the monthly transition of resistance ratios with regard to the specific wards that have the moving lines of inpatients in order to verify the hypothesis that resistant strains may be carried from ward to ward along the moving lines of inpatients.
\end{abstract}

Results: The data of 2,113 Staphylococcus aureus strains were accumulated and analyzed. Overall Oxacillin resistance ratio in our hospital was 65.7\%. The ward of the smallest Oxacillin resistance ratio was Pediatrics/Ophthalmology ward. The ratios of Oxacillin resistant were varied as from $67.9 \%$ to $96.7 \%$ regardless the categories of wards such as internal medicine or surgery. Multi-resistant MRSA strains were overwhelmingly dominant in the wards of surgery. The ratios of the Gentamicin sensitive strains that were resistant to Oxacillin were high over the every ward. The moving lines of inpatients existed between ICU/CCU ward and three rear wards. Two rear wards whose Oxacillin resistance changes were reflected to those of ICU/CCU, but one rear ward was not.

Conclusion: Variation of resistant degree among wards were very obvious and large. We could survey the wards where patient-to-patient transmission of resistant organisms might occur along the moving lines of inpatients. WHONET 5 will be recognized as an analysis and surveillance tool for every infection control team to survey the suspicious wards.

(J Nippon Med Sch 2004; 71: 345-351)

Key words: WHONET 5, Staphylococcus aureus strains, MRSA, resistance profile, infection control

Correspondence to Toru Mochizuki, MD, PhD., Department of Emergency and Critical Care Medicine, Nippon Medical School Second Hospital, 1-396 Kosugi-cho, Nakahara-ku, Kawasaki, Kanagawa 211-8533, Japan

E-mail :moti@nms.ac.jp

Journal Website (http://www.nms.ac.jp/jnms/) 


\section{Introduction}

The hospital microbiology laboratory provides high quality microbiological testing reports as a daily task. The information is crucial evidence based data for the nosocomial infection control'. The World Health Organization has established the implemented surveillance tool for antimicrobial resistance that is known WHONET $^{1-3}$. We analyzed our hospital laboratory microbiological data by using WHONET 5 that is a computer software that can accumulate and analyze laboratory microbiological data in order to put them to clinical $u^{2 e^{2.3}}$, and acquired information of antimicrobial resistance of Staphylococcus aureus among every ward in our hospital.

\section{Objective}

The purpose of this study is to acquire the information of antimicrobial resistance of Staphylococcus aureus strains among every ward by WHONET 5, and to enlighten the usefulness of WHONET 5 as an analysis and surveillance tool for antimicrobial resistance.

\section{Materials and Methods}

\section{Materials.}

The database of Staphylococcus aureus from every ward of Nippon Medical School Chiba Hokusoh Hospital, from September 2001 till December 2002.

\section{Methods.}

1) Analysis was performed under the condition as one isolate per one patient. Starting of 'resistance profile' analysis in WHONET 5 and analyzing the microbiological laboratory testing reports for every ward (15 clinical departments) were performed. We have 13 wards including ICU/CCU, and abbreviate 'the second floor west ward' to ' $2 \mathrm{~W}$ ' for example hereafter.

2) We chose Oxacillin, Levofloxacin , Erythromycin and Gentamicin as the antimicrobials that need to be investigated for resistance, because these four categories of antimicrobials are essential to current treatment. When the resistance profile analysis of oxacillin was activated, the resistant strains are indicated ' $\mathrm{O}$ ' as its initial, the Levofloxacin resistant strains are indicated ' $L$ ', the Erythromycin resistant strains are indicated ' $\mathrm{E}$ ' and the Gentamicin resistant strains are indicated ' $G$ ', respectively. In case of multi-resistant to Oxacillin, Levofloxacin, Erythromycin and Gentamicin, the indication is 'OLEG'.

3) We verified the hypothesis that resistant strains may be carried from ward to ward along the moving lines of inpatients. As its method, we evaluated the monthly transition of resistance ratios with regard to the specific wards that have the moving lines of inpatients.

\section{Results}

1) The data of 2,113 Staphylococcus aureus strains were accumulated and analyzed by WHONET 5 . Overall Oxacillin resistance ratio in our hospital was $65.7 \%$. We indicated resistance ratio of each ward to Oxacillin, resistance profile and ratio (Table $\mathbf{1}$ ).

(1) Comparison of resistance to Oxacillin among all wards

The ward of the smallest Oxacillin resistance ratio was 2E (Pediatrics/Ophthalmology ward) and the number was $21.0 \%$, followed by the ratio was $40 \%$ at $4 \mathrm{E}$ (Obstetrics/Gynecology ward). Regardless of the categories of wards such as internal medicine or surgery, the ratios of Oxacillin resistant were varied as from $67.9 \%$ till $96.7 \%$ excluding $2 \mathrm{E}$ and $4 \mathrm{E}$. Variance of resistant degree among wards were very obvious and large as shown in Table 1.

(2) Resistance profile of Oxacillin resistant strains

The ward of the largest ratio of resistance profile that indicated OLEG was 5W (Surgery ward) and the number was $51.8 \%$, followed by the ward $4 \mathrm{~W}$ (Obstetrics/Gynecology/Urology/Surgery ward) with the number of $32.7 \%$. The ratios of resistance profile that indicated OLEG of the rest wards were less than $10 \%$.

(3) Ratios of the Gentamicin sensitive strains that were resistant to Oxacillin among all wards

The ward of the largest ratio of the Gentamicin 
Table 1 Resistance profiles on Staphylococcus aureus strains among all wards

The data of 2,113 Staphylococcus aureus strains were accumulated and analyzed for the Oxacillin resistance, Levofloxacin resistance, Erythromycin resistance and Gentamicin resistance by using WHONET 5. Overall Oxacillin resistance ratio in our hospital was $65.7 \%$. We indicated resistance ratio of each ward to Oxacillin, resistance profile and ratio.

\begin{tabular}{l|rrrrrrrrrrrrr}
\hline & ICU/CCU & $2 \mathrm{E}$ & $2 \mathrm{~W}$ & $3 \mathrm{E}$ & $3 \mathrm{~W}$ & $4 \mathrm{E}$ & $4 \mathrm{~W}$ & $5 \mathrm{E}$ & $5 \mathrm{~W}$ & $6 \mathrm{E}$ & $6 \mathrm{~W}$ & $7 \mathrm{E}$ & $7 \mathrm{~W}$ \\
\hline 0 total & 67.9 & 21.0 & 80.7 & 96.7 & 83.0 & 40.0 & 93.1 & 93.6 & 84.8 & 89.1 & 72.3 & 87.8 & 85.4 \\
\hline 0 & & & & & & 20.0 & & & & & & 0.6 & \\
$0 \quad \mathrm{G}$ & 0.7 & 3.9 & 2.9 & & & & & & & & & & \\
0 EG & & 6.2 & & 0.6 & 3.8 & & & & & & & 5.3 & \\
0L G & & & & & 5.1 & & 8.2 & & & & & & \\
0 E & & & & & & & & 0.9 & & & & & \\
0LE & 50.7 & 9.3 & 70.0 & 87.5 & 57.6 & 20.0 & 52.2 & 90.9 & 33.0 & 85.0 & 63.1 & 62.9 & 80.5 \\
0LEG & 16.5 & 1.6 & 7.8 & 8.6 & 16.5 & & 32.7 & 1.8 & 51.8 & 4.1 & 9.2 & 19.0 & 4.9 \\
\hline
\end{tabular}

Percent indications (\%) on each ward Resistance profiles on 2,113 strains O: Oxacillin resistance OL: Oxacillin and Levofloxacin resistance OEG: Oxacillin and Erythromycin and Gentamicin resistance OLE: Oxacillin and Levofloxacin and Erythromycin resistance OLEG: Oxacillin and Levofloxacin and Erythromycin and Gentamicin resistance

Table 2 Ratios of Gentamicin sensitive strains which are Oxacillin resistant among all wards

\begin{tabular}{l|ccccccccccccc}
\hline & ICU/CCU & $2 \mathrm{E}$ & $2 \mathrm{~W}$ & $3 \mathrm{E}$ & $3 \mathrm{~W}$ & $4 \mathrm{E}$ & $4 \mathrm{~W}$ & $5 \mathrm{E}$ & $5 \mathrm{~W}$ & $6 \mathrm{E}$ & $6 \mathrm{~W}$ & $7 \mathrm{E}$ & $7 \mathrm{~W}$ \\
\hline $\begin{array}{l}\text { 0 total } \\
\begin{array}{l}\text { G sen- } \\
\text { sitive }\end{array}\end{array}$ & 67.9 & 21.0 & 80.7 & 96.7 & 83.0 & 40.0 & 93.1 & 93.6 & 84.8 & 89.1 & 72.3 & 87.8 & 85.4 \\
\hline
\end{tabular}

O: Oxacillin G: Gentamicin

sensitive strains that were resistant to Oxacillin was $4 \mathrm{E}$ and the number was $100 \%$. The wards of the ratio more than $90 \%$ were $5 \mathrm{E}$ (Digestive/Surgery ward) and the number was 98.1\%, 6E (Internal medicine (Kidney, Endocrine)) and the number was 95.4\% , 7W (Otorhinolaryngology/Dermatology / Mental health ward) and the number was $94.3 \%, 4 \mathrm{~W}$ and the number was $93.1 \%$ and 3E (Internal medicine (Circulation)/Thoracic surgery ward) and the number was $90.5 \%$.

The ward of the smallest ratio was $5 \mathrm{~W}$ and the number was $38.9 \%$, and the ward of the second smallest ratio was $2 \mathrm{E}$ and the number was 44.3

(Table 2).

2) Comparison of Oxacillin resistance changes monthly among ICU/CCU and the rear wards of $\mathrm{ICU} / \mathrm{CCU}$

Inpatients are admitted directly from the outpatients sections or the emergency room as well as scheduled, and basically, there are no transference of inpatients among wards. There are three rear wards $(2 \mathrm{~W}, 3 \mathrm{E}$ and $3 \mathrm{~W}$ ) of ICU/CCU. Transference of inpatients exists among ICU/CCU and $2 \mathrm{~W}, 3 \mathrm{E}$ and $3 \mathrm{~W}$. The moving lines of inpatients are between ICU/CCU and $2 \mathrm{~W}, 3 \mathrm{E}$ and $3 \mathrm{~W}$, respectively (Fig. 1).

Comparion of the Oxacillin resistance changes and the ratio changes of OLEG monthly among rear wards of ICU/CCU was presented at Fig. 2.

There was a tendency that when the Oxacillin resistance ratios of ICU/CCU were high, simultaneously the Oxacillin resistance ratios of $2 \mathrm{~W}$ and $3 \mathrm{~W}$ were high, and conversely when the Oxacillin resistance ratios of ICU/CCU were low, simultaneously the Oxacillin resistance ratios of $2 \mathrm{~W}$ and $3 \mathrm{~W}$ were low. Resistant strains may be carried from ward to ward along the moving lines of inpatients was suspected, because the Oxacillin resistance ratio changes of ICU/CCU among every month might be reflected by those changes of rear 


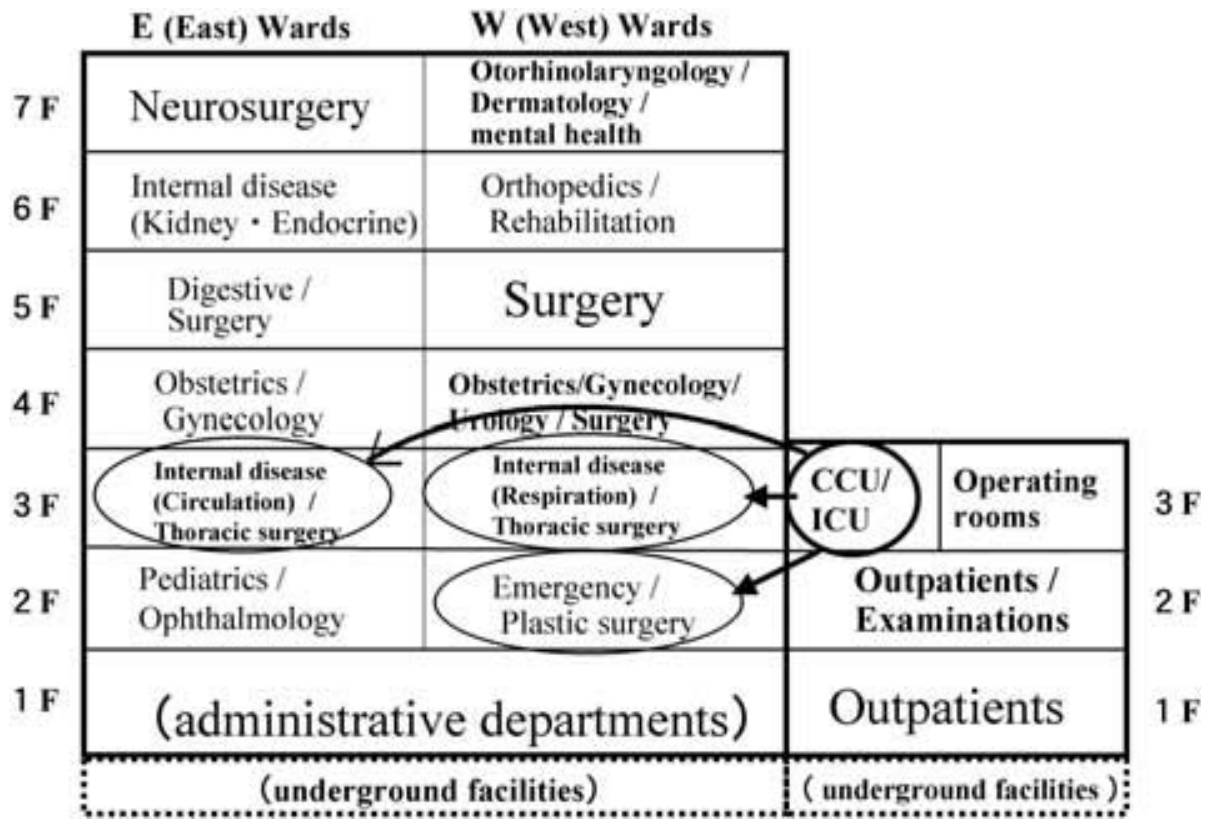

Fig. 1 Transference of inpatients within our hospital

Transference of inpatients within our hospital exists among ICU/CCU and $2 \mathrm{~W}, 3 \mathrm{E}$ and $3 \mathrm{~W}$. The moving lines of inpatients are between ICU/CCU and $2 \mathrm{~W}, \mathrm{ICU} / \mathrm{CCU}$ and $3 \mathrm{E}$ and ICU/CCU and $3 \mathrm{~W}$ respectively.

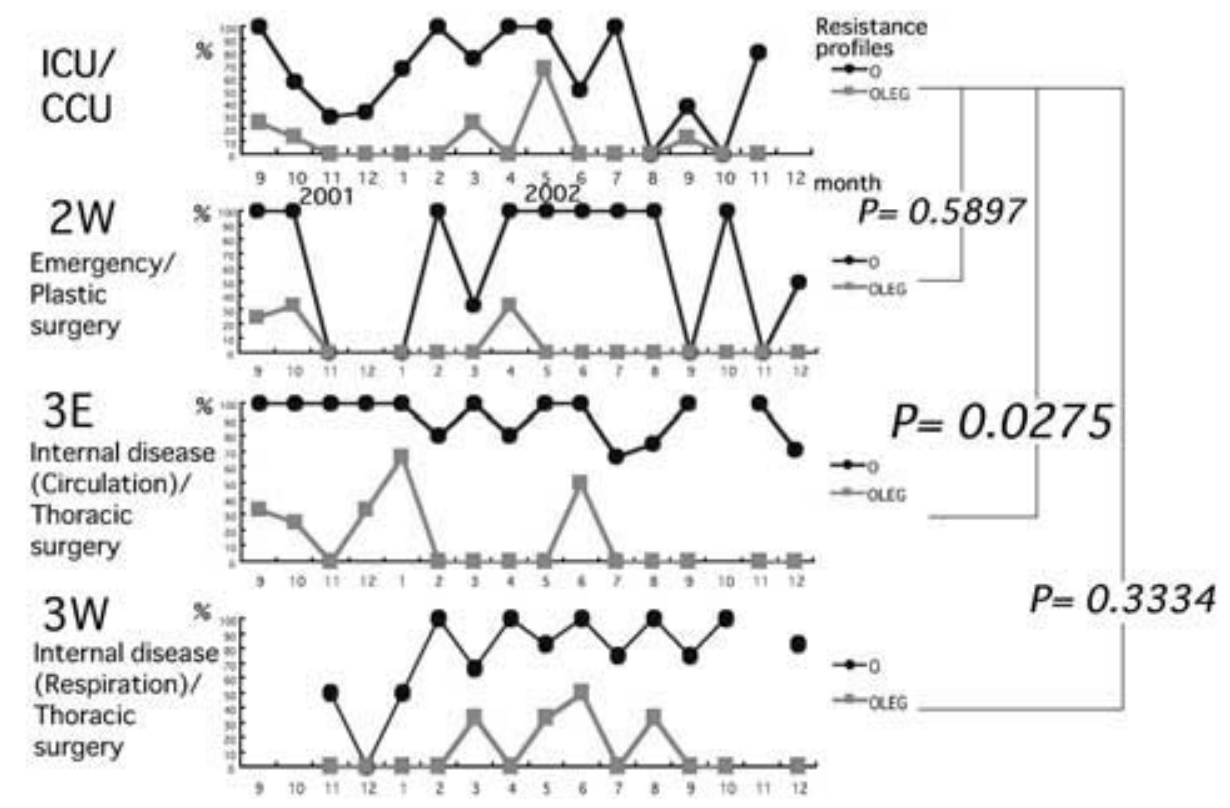

Fig. 2 Compulsion of Oxacillin resistance changes monthly among ICU/CCU and the rear wards of ICU/CCU

Compulsion of the Oxacillin resistance changes and the ratio changes of OLEG monthly among rear wards of ICU/CCU was presented as the graph. The ratios of Oxacillin resistance were indicated as closed circles and those of OLEG were indicated as closed squares in the graph.

There was statistically significant difference between ICU/CCU and 3E and the number of $p$-value was 0.0275 . There were no statistically significant difference between ICU/CCU and $2 \mathrm{~W}$, ICU/CCU and $3 \mathrm{~W}$, and the number of $p$-value was $0.5897,0.3334$ respectively. 
Table 3 The list of antimicrobials that can be evaluated by WHONET 5

The antimicrobials that can be evaluated by WHONET 5 in our hospital are listed and the number is 44 .

Penicillin G, Ampicillin, Oxacillin, Piperacillin, Amoxicillin/Clavulanic acid, Ampicillin/Sulbactam

Cefaclor, Cefazolin, Cefmetazole, Cefotaxime, Cefotiam, Cefpirome, Ceftazidime, Flomoxef, Cefozopran, Cefdinir,

Cefepime, Cefditoren, Cefixime, Cefriaxone, Cefamandole, Cefoperazone, Cephalothin

Imipenem, Meropenem

Aztreonam

Amikacin, Gentamicin, Arbekacin, Isepamicin, Tobramycin

Levofloxacin, Ciprofloxacin

Erythromycin, Clarithromycin

Minocycline, Tetracycline

Teicoplanin, Vancomycin

Clindamycin

Trimethoprim/Sulfamethoxazole

Chloramphenicol

Rifampin

Fosfomycin

44 antimicrobials

wards such as $2 \mathrm{~W}$ and $3 \mathrm{~W}$. The analysis of the monthly Oxacillin resistance ratio changes between $\mathrm{ICU} / \mathrm{CCU}$ and $2 \mathrm{~W}$, ICU/CCU and $3 \mathrm{E}$ and ICU/CCU and $3 \mathrm{~W}$ using Mann-Whitney $\mathrm{U}$ test indicated the statistically, with significant difference between ICU/CCU and 3E and the p-value 0.0275. There were no statistically significant difference between ICU/ $\mathrm{CCU}$ and $2 \mathrm{~W}, \mathrm{ICU} / \mathrm{CCU}$ and $3 \mathrm{~W}$, and p-value was $0.5897,0.3334$, respectively.

\section{Discussion}

WHONET 5 is a computer software that can accumulate and analyze enormous laboratory microbiological data from the hospital microbiology laboratory in order to put them to clinical use $\mathrm{u}^{2.3}$. This software was authored by John M. Stelling and Thomas F. O'Brien in 1997 and developed up to version 5 (WHONET 5) at present ${ }^{1.3}$. WHONET 5 has the ability to analyze for 335 species of organisms totally and 7 groups of organisms (for example 'All gram-positive organisms'). The antimicrobials that can be evaluated by WHONET 5 in our hospital are listed as Table 3. The WHONET can perform several types of analysis, and the analysis type should be chosen in the time of data output (Fig. 3) . WHONET 5 has 6 analysis types. In this study, we chose the 'resistant profile' analysis.

We chose Staphylococcus aureus strains, because those are the targets for the nosocomial infection control. The next, we chose antimicrobials of different categories in addition to Oxacillin. We chose Levofloxacin as the representatives for Fluoroquinolone's groups, Erythromycin for Macrolide's groups and Gentamicin for Aminoglycoside's groups 5 .

The number $65.7 \%$ of the overall Oxacillin resistance ratio in our hospital seems to be comparatively high. The clinical training hospitals in Japan have been slightly above or equal to $50 \% \%^{6,7}$.

We evaluated multi-resistant MRSA ratios among all wards. We obtained the fact that the highest 3 wards were Surgery ward, Obstetrics/Gynecology/ Urology/Surgery ward and Neurosurgery ward. This indicated that multi-resistant MRSA might be overwhelmingly dominant in the wards of surgery category. An overdose use of antimicrobials should target to the skin surface pathogenic organisms such as Staphylococcus aureus strains. This would result in the emergence of creating a lot of resistant strains. The resistant profiles among all wards were seen to indicate that the ratios of Aminoglycosides sensitive strains might be high ${ }^{5}$. According to this fact, it will be considered as sufficient to use Aminoglycoside's groups together with Penicillin's groups or low generation Cephalosporin's groups on the antimicrobial therapy for Staphylococcus aureus strains. and these choices will improve current 


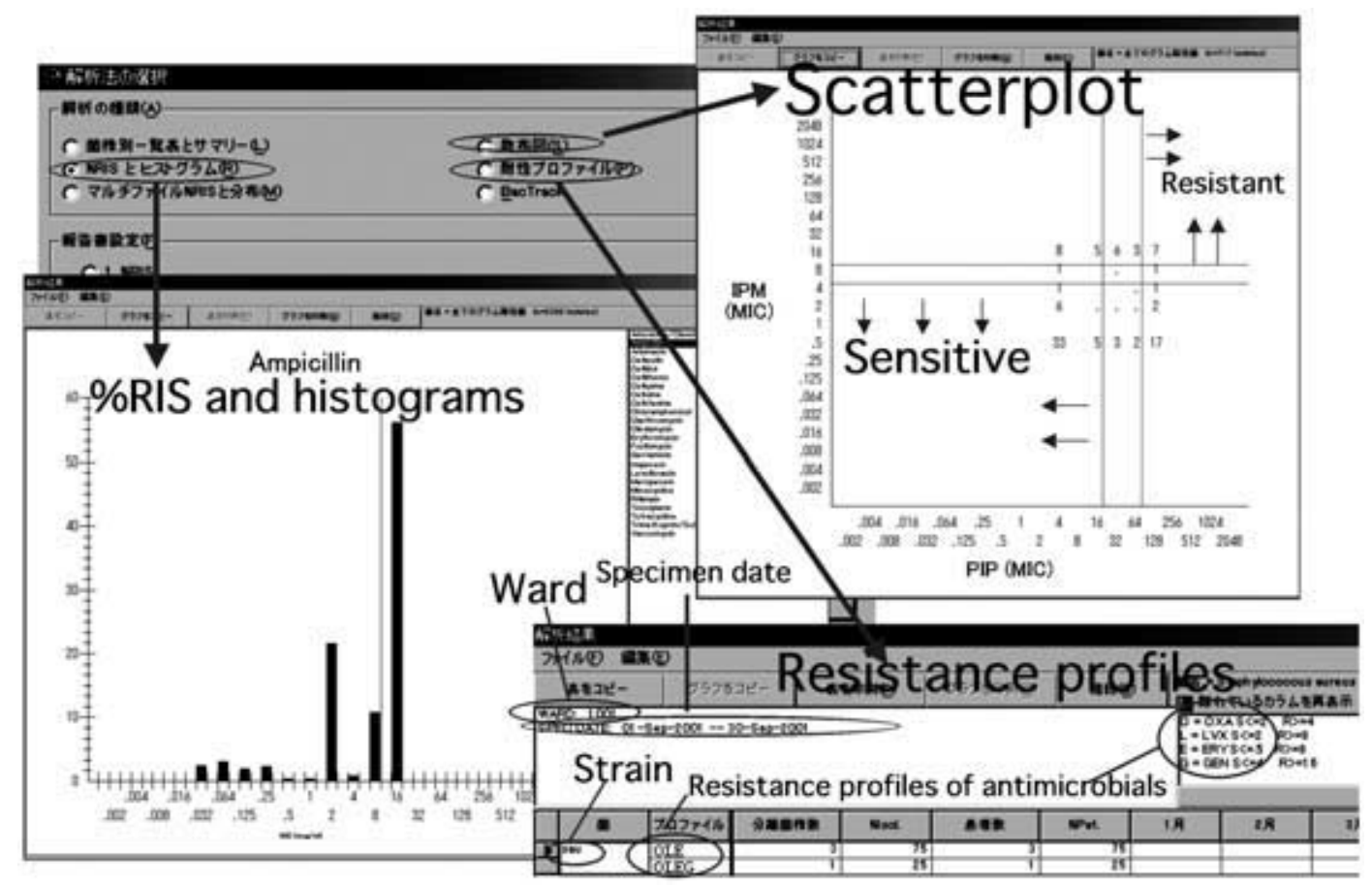

Fig. 3 Data analysis on WHONET

According to the desired information, the analysis type should be chosen in the time of data output. WHONET 5 has 6 analysis types as 'Isolate listing and summary', '\%RIS (R: Resistant, I: Intermediate, S: Sensitive) and histograms', 'Multi-File \%RIS and distributions', 'Scatterplot', 'Resistant profiles' and 'Bac Track'. In this study, we chose and used the 'resistant profile' analysis, because we want to acquire the information of antimicrobial resistance to the specific antimicrobials.

situation to both misuse and overuse of the strong and very broad spectrum antimicrobials ${ }^{8.9}$.

$\mathrm{ICU} / \mathrm{CCU}$ is a mixed ward that consists of the department of emergency and critical care medicine, and post-operative patients of thoracic surgery with cardiopulmonary machine. Most of these patients are compromised hosts. Usually strong and broadspectrum antimicrobials have been chosen from the beginning. Paterson commented that ICUs are frequently a hospital's epicenter for antimicrobial resistance. In part, this is because of the widespread use of broad empiric therapy. A second major contributor is the close monitoring of all laboratory data in a critically ill patients and a tendency to "treat" all positive cultures with antimicrobials, regardless of whether those cultures are associated with colonization or infection. Third, the high demands on nursing and medical personnel in ICUs would worsen the possibility for patient-to-patient transmission of multi-resistant organisms. The usual vehicle for transmission is the hands of healthcare workers ${ }^{10}$. Therefore, we assumed the hypothesis that resistant strains may be carried from ward to ward along the moving lines of inpatients. The monthly Oxacillin resistance ratio changes in ICU/ CCU might be reflected by those changes of rear wards, and it suggested the possibility that resistant strains may be carried from ward to ward by patient-to-patient transmission among these wards along the moving lines of inpatients (Fig. 2).

The moving lines of inpatients are found between ICU/CCU ward and three rear wards. However two rear wards had paralled Oxacillin resistance changes with those of ICU/CCU, but one rear ward did not.

In this point, we suggest an another factor that the difference of implementation of infection control such as "standard precaution" among wards might influence these results. It is considerd that health care workers working at wards which are suspicious to transmit multi resistant-bacteria from patient-to- 
patient don't have sufficient information on drugresistant bacterias. Such information should be given. WHONET 5 will be recognized as an analysis and surveillance tool for an infection control team.

This time we reported only an analysis of resistant profiles about Staphylococcus aureus strains. We should survey continuously other organisms that need to be investigated by WHONET 5 . This antimicrobial sensitivity and resistance information will be crucial evidence based data for "appropriate use of antimicrobials".

\section{Conclusion}

1) It was clear that the ratios of the Oxacillin resistance were varied by each ward regardless of the categories of wards such as internal medicine or surgery.

2) Multi-resistant MRSA strains were overwhelmingly dominant in the wards of surgery category.

3) It was found that the ratios of the Gentamicin sensitive strains that were resistant to Oxacillin were high over the wards. The choice of Aminoglycoside's groups together with Penicillin's groups or low generation Cephalosporin's groups will be alternative to strong and broad spectrum antimicrobials.

4) We could survey the wards where patient-topatient transmission of resistant organisms might occur along the moving lines of inpatients.

WHONET 5 will be an analysis and surveillance tool for an infection control team to survey the suspicious wards.

Beyond that we should survey continuously other organisms for “appropriate use of antimicrobials".

\section{References}

1. World Health Organization: WHO global strategy for containment of antimicrobial resistance: 2001.2; $\mathrm{WHO} / \mathrm{CDS} / \mathrm{CSR} / \mathrm{DRS}$.

2. World Health Organization: WHONET5 Microbiology Laboratory Database Software: 1999. 1; WHO/CDS/ $\mathrm{CSR} / \mathrm{DRS}$

3. O’Brien TF, Eskildsen MA, Stelling JM: Using internet discussion of antimicrobial susceptibility databases for continuous quality improvement of the testing and management of antimicrobial resistance. Clinical infectious diseases 2001; 33 (Suppl 3): S118123.

4. Tenover FC, Mohammed MJ, Stelling JM: Ability of laboratories to detect emerging antimicrobial resistance: Proficiency testing and quality control results from the World Health Organization's external quality assurance system for antimicrobial susceptibility testing. Journal of clinical microbiology 2001; 39: 241-250.

5. Oteo J, Cruchaga S, Campos J, Saez JA, Baquero F: Antibiotic resistance in blood isolates of Staphylococcus aureus in 31 Spanish hospitals participating in the European Antimicrobial Resistance Surveillance System (2000). Med Clin (Barc) Spanish. 2002; 119: 361-365.

6. Kakinohana S, Uemura E, Insisiengmay S: Staphylococcus aureus Isolated from hospital staff: a comparative study of Laos and Japan. Journal of Infection and chemotherapy 2002; 8: 336-340.

7. Osawa K, Baba C, Ishimoto T: Significance of methicillin-resistant Staphylococcus aureus (MRSA) survey in a university teaching hospital. Journal of Infection and chemotherapy 2003; 9: 172-177.

8. Pujol M, Gudiol F: Evidence for antibiotic cycling in control of resistance. Current Opinion in Infectious Diseases 2001; 14: 711-715.

9. Kollef MH, Ward S, Sherman G: Inadequate treatment of nosocomial infection is associated with certain empiric antibiotic choices. Critical Care Medicine 2000; 28: 3456-3464.

10. Paterson DL, MBBS, FRACP: Restrictive antibiotic policies are appropriate in intensive care units. Critical Care Medicine 2003; 31 (Supplement): S25S28. 